\title{
Inhibition of tumor growth through suppression of angiogenesis by brain-specific angiogenesis inhibitor 1 gene transfer in murine renal cell carcinoma
}

\author{
SHIGETAKA KUDO ${ }^{1}$, RYUICHIRO KONDA ${ }^{1}$, WATARU OBARA ${ }^{1}$, DAISUKE KUDO ${ }^{1}$, \\ KENZABURO TANI $^{2}$, YUSUKE NAKAMURA ${ }^{3}$ and TOMOAKI FUJIOKA ${ }^{1}$ \\ ${ }^{1}$ Department of Urology, Iwate Medical University School of Medicine, Morioka; ${ }^{2}$ Division of Molecular and \\ Clinical Genetics, Medical Institute of Bioregulation, Kyushu University Hospital, Kyushu University, \\ Fukuoka 812-8582; ${ }^{3}$ Laboratory of Molecular Medicine, Human Genome Center, Institute of \\ Medical Science, The University of Tokyo, Tokyo 108-8639, Japan
}

Received March 17, 2007; Accepted April 26, 2007

\begin{abstract}
This study was designed to elucidate the therapeutic effect of transfering the brain-specific angiogenesis inhibitor 1 (BAI1) gene to a mouse renal cell carcinoma cell line (Renca). Female BALB/c mice were inoculated subcutaneously with wild-type Renca (Renca/Wild) cells or Renca cells transfected with the BAI-1 (Renca/BAI-1) or LacZ (Renca/LacZ) gene. Tumor growth was observed every other day from 3 to 35 days after implantation. Moreover, the intratumoral injection of the adenovirus vector containing the gene encoding BAI1 was conducted at two-day intervals from 11 to 31 days after implantation of the Renca/Wild or Renca/BAI1 tumor. Tumor blood flow was measured by colorimetric angiogenesis assay (CAA). The concentration of the vascular endothelial growth factor (VEGF) in the cell culture supernatants was determined by enzyme-linked immunoassay. The size of the Renca/BAI1 tumor was significantly $(\mathrm{p}<0.01)$ suppressed compared to the Renca/ Wild and Renca/LacZ tumors 21 days after tumor implantation. The injection of the BAI1 viral vector at 2-day intervals significantly inhibited the growth of both the Renca/Wild and Renca/BAI1 tumors. The blood volume measured by CAA and microvessel density was significantly lower in the Renca/BAI1 than in the Renca/Wild and Renca/LacZ tumors $(\mathrm{p}<0.01$ and $\mathrm{p}<0.05$, respectively). A
\end{abstract}

Correspondence to: Dr Ryuichiro Konda, Department of Urology, Iwate Medical University School of Medicine, 19-1 Uchimaru, Morioka 020-8505, Japan

E-mail: ryukonda@iwate-med.ac.jp

Abbreviations: BAI1, brain-specific angiogenesis inhibitor 1; RCC, renal cell carcinoma; TSP, thrombospondin; VEGF, vascular endothelial growth factor

Key words: gene therapy, angiogenesis, brain-specific angiogenesis inhibitor 1, renal cell carcinoma significant $(\mathrm{p}<0.01)$ reduction in VEGF concentration in the supernatant was demonstrated in the Renca/BAI1 compared with the Renca/Wild and Renca/LacZ cell cultures. These observations suggest that the transfer of the BAI1 gene to Renca can suppress the tumor growth via the inhibition of angiogenesis. The down-regulation of VEGF production in tumor cells contributes to this anti-tumor effect.

\section{Introduction}

New vascular formation is required for tumor growth, invasion and metastasis $(1,2)$. Various factors that regulate angiogenesis have been elucidated successively over the years, and cancer treatments that target these factors have been devised $(1,2)$. Radical nephrectomy or nephron-sparing surgery is the main-stay of curative treatment for localized renal cell carcinoma ( $\mathrm{RCC}$ ), which is unresponsive to radiotherapy and also refractory to chemotherapy (3). Immunotherapies using interferons and interleukin 2 have been used for advanced RCC. However, the two therapies alone as well as in conjunction have demonstrated disappointing success rates of $20 \%$ or less (4). RCC is a typical hypervascular tumor, and neovascularisation is thought to play a principal role in tumor progression (5). The inhibition of angiogenesis could lead to promising developments of new treatment modalities.

A brain-specific cancer-regulating p53 target gene was isolated and identified as a new angiogenesis inhibitor, and was named the brain-specific angiogenesis inhibitor 1 (BAI1) gene (6). The BAI1 gene encodes a 1584-amino-acid product containing five thrombospondin (TSP) type 1 repeats. A recombinant protein analogous to the TSP type 1 repeats of this gene product has been shown to inhibit angiogenesis induced by the basic fibroblast growth factor (bFGF) in the rat cornea (6). Although BAI1 mRNA was initially reported to be expressed specifically in the brain, this gene has subsequently been demonstrated in the neoplastic and/or nonneoplastic tissues of the colon, stomach and lung, and its expression correlates inversely with vascular density (7-9). 
The overexpression of BAI1 inhibited tumor growth by suppressing tumor angiogenesis in human pancreatic adenocarcinoma cells (Panc-1) (10). Moreover, the overexpression of BAI1 induced cell death in human umbilical vein endothelial cells $(10,11)$.

We designed this study in order to elucidate the possibility of gene therapy by BAI1 gene transfer in RCC. Gene transfer in a mouse RCC cell line (Renca) was conducted using replication-deficient recombinant adenoviral vectors encoding BAI1 (10). Tumor growth was observed after the subcutaneous inoculation of Renca cells transfected with the BAI1 gene and compared with wild-type cells and cells transfected with LacZ. In addition, we evaluated whether the anti-tumor effect of the BAI1 gene transfer is associated with the inhibition of new vascular formation.

\section{Materials and methods}

Reagents. A commercial MTT [3-(4,5-dimethylthiazol-2-yl)2,5-diphenyltetraslium bromide] colorimetry kit was purchased from Chemico (Temecula, CA, USA). X-gal staining solution was from Gibco BRL (Tokyo, Japan) and used at $37^{\circ} \mathrm{C}$. An RNeasy Mini Kit was purchased from Qiagen (Tokyo, Japan). The commercial ELISA kits (human bFGF ANALYZA ELISA and mouse VEGF ANALYZA ELISA) were from Genzyme (Minneapolis, USA).

Cell lines and animals. The mouse RCC cell line (Renca) was kindly provided by Dr R.H. Wiltrout (N.C.I., Fredrick, $\mathrm{MD}$ ), which was maintained in vivo by serial intrarenal passage. In vitro culture of the Renca cells was performed in RPMI-1640 containing $10 \%$ fetal cow serum (FCS) at $37^{\circ} \mathrm{C}$ under $5 \% \mathrm{CO}_{2}$ (13). Female, euthymic BALB/c mice were housed in a special pathogen-free facility and used routinely at 8 to 10 weeks of age when they weighed 22 to $24 \mathrm{~g}$.

Gene transfection and in vitro tumor growth. Gene transfer in the Renca cells was conducted using the replicationdeficient recombinant adenoviral vector encoding the full length cDNA of BAI1 (Renca/BAI1) or LacZ (Renca/LacZ), at a multiplicity of infection (MOI) of 20 for $72 \mathrm{~h}$ as described previously $(10,12)$. Renca cells not transfected with any gene (Renca/Wild) were used as the controls.

For the in vitro cytotoxicity test, Renca cells ( $1 \times 10^{5} /$ well) and the viral vector at an MOI of 1, 2, 10, 20, 50, 100, 200 and 300 were added to a 96-well microplate. After incubation for $72 \mathrm{~h}$, the MTT reagent was added and the cells were incubated for a further $4 \mathrm{~h}$. The reaction was then stopped by the addition of isopropanol hydrochloride, and the absorbance $(540 \mathrm{~nm})$ was measured.

In order to confirm the vector transfer efficiency, Renca cells were transfected with the control vector (AdexCA-LacZ) at an MOI of 20 for $72 \mathrm{~h}$ and $\beta$-galactosidase was assayed using X-gal staining. The Renca/LacZ cells were fixed in $2 \%$ formaldehyde and $0.2 \%$ glutaraldehyde in PBS(-) for $10 \mathrm{~min}$ at $4^{\circ} \mathrm{C}$. The fixative was removed, and then $\mathrm{X}$-gal staining solution was added. After $3 \mathrm{~h}$ of reaction at $37^{\circ} \mathrm{C}$, the cells were examined under a light microscope.

In order to measure the in vitro cellular proliferation capability, Renca cells $\left(1 \times 10^{5}\right)$ inoculated in a $25 \mathrm{~cm}^{2}$ tissue culture flask were transfected with the virus vector (AdexCALacZ or pAdex1Cawt-BAI1) at an MOI of 20, and cell counts were performed on days $1,3,5$ and 7 .

Confirmation of the BAIl gene transfer using RT-PCR. RNA was extracted from Renca/Wild, Renca/LacZ and Renca/ BAI1 cells using the RNeasy Mini Kit, and subjected to RT reaction using oligo(dT)12-18 and SuperScript II (Gibco $\mathrm{BRL})$ at $37^{\circ} \mathrm{C}$ for $90 \mathrm{~min}$. PCR was then performed using Taq Gold DNA polymerase (Applied Biosystems, Tokyo, Japan) and the primers given below. The thermal conditions were $95^{\circ} \mathrm{C}$ for $10 \mathrm{~min}$, followed by 35 cycles $\left(94^{\circ} \mathrm{C}\right.$ for $30 \mathrm{sec}$, $55^{\circ} \mathrm{C}$ for $30 \mathrm{sec}$, at $72^{\circ} \mathrm{C}$ for $60 \mathrm{sec}$ ), and a final extension at $72^{\circ} \mathrm{C}$ for $10 \mathrm{~min}$. The PCR products were electrophoresed on a $1.5 \%$ agarose gel, stained with ethidium bromide, and examined under UV light. The primers used were G3PDH, 5'-TGAAGGTCGGTGTGAACGGATTTGGC-3' (sense) and 5'-CATGTAGGCCATGAGGTCCACCAC-3' (anti-sense); BAI1 (6), 5'-ACTCATCCTGCGACGGTGTG-3' (sense, E1S) and 5'-TCCCTCAGGTCCTTCATGCG-3' (anti-sense, E1A). The RT-PCR products obtained in the above manner were fluorescent dyed using the BigDye Terminator (Applied Biosystems), and the base sequences were determined using the ABI PRISM310 Genetic Analyser.

Assay of bFGH and VEGF in cell culture supernatant. Supernatants were obtained from the Renca/Wild, Renca/ LacZ and Renca/BAI1 $(n=5)$ cell cultures on days 1, 3, 5 and 7. Concentrations of bFGF and the vascular endothelial growth factor (VEGF) were determined using the commercial ELISA kits mentioned above.

In vivo tumor growth. Renca/Wild, Renca/LacZ or Renca/ BAI1 cells $\left(1 \times 10^{6}\right.$ cells in $\left.0.05 \mathrm{ml}\right)$ were inoculated subcutaneously into the dorsal skin of mice $(n=10)$. The tumors were observed on consecutive days for 35 days, and the long and short diameters of the tumors were measured. Tumor volume was estimated according to the following formula, and used as a measure of the antitumor effects: Tumor volume $\left(\mathrm{mm}^{3}\right)=(\text { short diameter })^{2} \times($ long diameter $) \times 0.5(13)$.

In order to examine the changes in BAI mRNA expression during the experimental period, we obtained Renca/BAI1 tumor tissues on days $3,5,9,15,21,29$. RNA was extracted from these tissues and subjected to RT-PCR using the same method used for the cultured Renca cells.

Tumor angiogenesis assay. Tumor neovascularisation activity and intratumor blood volume was determined using in vivo colorimetric angiogenesis assay (CAA) (13). Renca/Wild, Renca/LacZ or Renca/BAI1 cells $\left(2 \times 10^{6}\right.$ cells in $0.05 \mathrm{ml}$ per site) were inoculated subcutaneoulsy into 4 sites of the dorsal skin of a mouse ( $\mathrm{n}=8$ per group). After $72 \mathrm{~h}$, $0.25 \mathrm{ml} 1 \%$ Evan's blue solution was injected into the caudal vein, and the tumors were removed 2 min later. The specimens of these tumors were dissolved in $0.35 \mathrm{ml}$ sodium sulfate/acetone, and the absorbance $(620 \mathrm{~nm})$ of the supernatant thus obtained was measured with an ELISA reader. Mouse blood collected after the injection of Evan's blue was dissolved in the same solvent and serially diluted. The blood volume was then calculated using the absorbance of the 


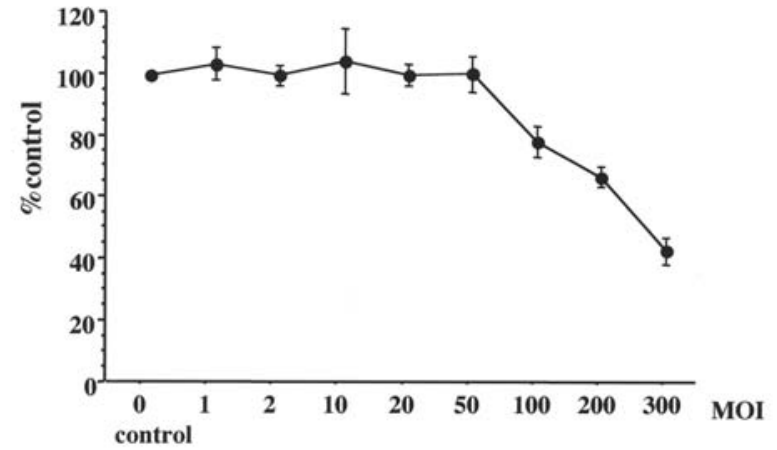

b

BAI1

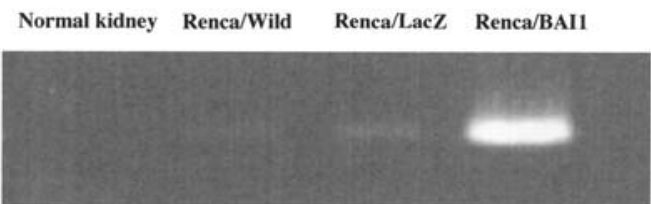

G3PDH

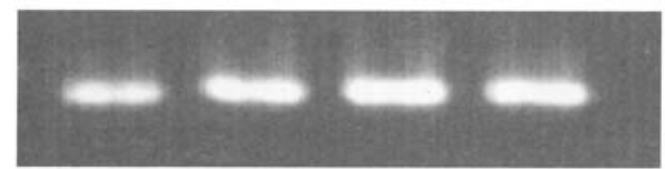

452bp

Figure 1. (a) In vitro cytotoxic effect of the viral vector at various multiplicities of infection (MOI) on the Renca cell culture. MTT assay was performed in duplicate. No cytotoxicity was observed when virus vectors were added at MOI 50 or less. (b) RT-PCR of the three Renca cell lines and normal kidney tissue. The expression of BAI1 was detected only in the Renca cells transfected with BAI1.

supernatant obtained from serially diluted mouse blood samples as the standard. The total blood volume of 2 transplant sites was defined as the tumor neovascularisation activity. Normal samples were obtained from non-inoculated sites in order to measure the blood volume in the non-tumor regions.

Immunohistochemistry and quantification of microvessel density. Immunostaining for CD31 was performed on 3-5 $\mu \mathrm{m}$ sections from paraffin-embedded tumor tissues $(n=6)$ on day 15 using rat anti-mouse CD31 (BD Pharmingen, San Diego, USA).

Intratumoral microvessel density (MVD) was determined by light microscopy after immunostaining the sections with anti-CD31 antibody. The MVD was expressed as the average number of the 5 highest areas identified within a single $\mathrm{x} 200$ per fields.

Intratumor administration of BAIl gene vector. Twenty microliters of pAdex1Cawt-BAI1 (1.7x10 $\left.10^{8} \mathrm{pfu} / \mathrm{ml}\right)$ or PBS(-) were injected into the subcutaneously implanted Renca/Wild or Renca/BAI1 tumor, starting on the 11th day after implantation and continuing on every other day for a total of 11 doses ( $\mathrm{n}=10$ for each group). Tumor volume was estimated using the same formula as described above.

Statistical analysis. The Mann-Whitney U test was used for the statistical analysis of the comparative data. Values of $\mathrm{p}<0.05$ were considered significant.

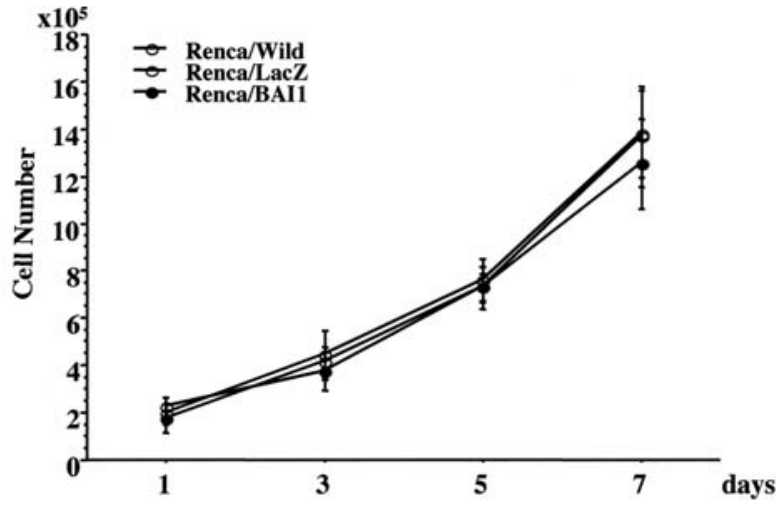

Figure 2. In vitro proliferation capability of Renca/Wild, Renca/LacZ and Renca/BAI1 cells. No significant difference was observed among the three cell lines.

\section{Results}

In vitro tumor growth. No cytotoxicity was observed by the addition of the virus vectors at MOI 50 or less, but toxicity was seen at MOI 100 or higher (Fig. 1a). Therefore, subsequent viral vector transfection experiments were performed using an MOI of 20 in order to eliminate the possibility of vector cytotoxicity. Beta-galactosidase staining of the Lac $\mathrm{Z}$ transfectants (MOI 20) confirmed a very high infection rate (almost 100\%) of tumor cells by adenovirus-mediated transfer. The results of RT-PCR of the RNA samples obtained from normal mouse kidney tissues $(n=3)$ and the three Renca cell lines are shown in Fig. 1b. A strong 453 bp band was seen only in the RNA sample of Renca/BAI1 cells, signifying that the BAI1 gene was transferred to the Renca cells. The base sequence for the PCR products obtained from the Renca/ BAI1 cells exactly matched that for the human BAI1 gene (6).

Fig. 2 shows the in vitro proliferation capability of the Renca/Wild, Renca/LacZ and Renca/BAI1 cells. The cell counts increased $\sim 7$-fold in all three cell cultures by the 7 th day. No significant difference in the proliferation rate was found among the Renca/Wild, Renca/LacZ and Renca/BAI1 cells.

Concentrations of bFGF and VEGF in cell culture supernatant. Assays of angiogenic factors in the cell culture supernatant are shown in Fig. 3 ( $n=5$ for each group). VEGF levels on days 3, 5 and 7 post-infection with the viral vector were significantly $(\mathrm{p}<0.01)$ lower in the Renca/BAI1 cells than in the Renca/Wild or Renca/LacZ cells (Fig. 3a). In contrast, no significant differences in the bFGF level were observed among the three cell lines throughout the experimental period (Fig. 3b).

In vivo tumor growth and angiogenesis. Renca/Wild and Renca/LacZ tumors demonstrated rapid growth, as shown by the tumor volume measured up to day 35 (Fig. 4a). In contrast, the growth of the Renca/BAI1 tumor was significantly $(\mathrm{p}<0.01)$ suppressed from days 27 to 35 compared with the Renca/Wild or Renca/LacZ tumor. The expression of BAI1 mRNA was maintained in the Renca/BAI1 tumors until day 15. However, the attenuation of BAI1 mRNA expression was observed on days 21 and 29 (Fig. 4b). 


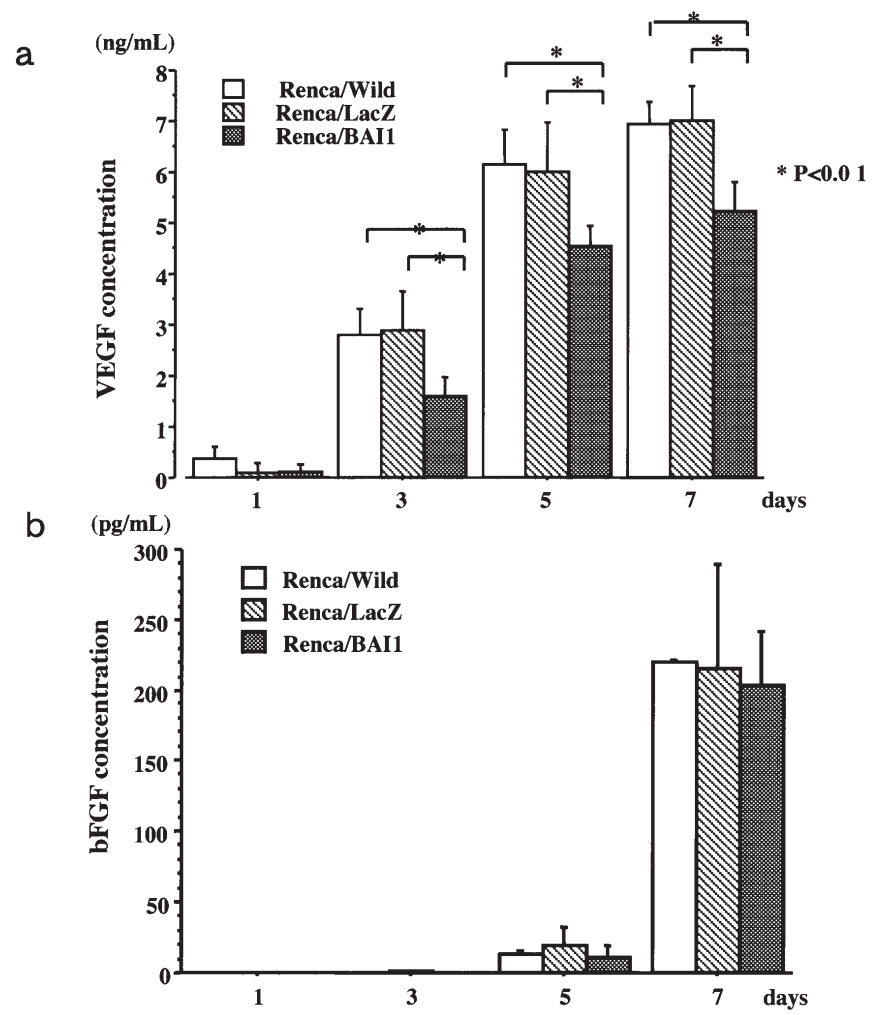

Figure 3. Concentrations of VEGF (a) and bFGF (b) in the cell culture supernatants. The levels of VEGF were significantly lower in the Renca/ BAI1 cell culture than in the Renca/Wild and Renca/LacZ cell cultures on days 3, 5 and 7 post-infection (a). No significant differences in the level of bFGF were noted among the three cell cultures (b).

Intratumor blood volumes as measured by the CAA method are shown in Fig. 5a. The blood content (mean \pm standard deviation; in $\mu 1 / 66.4 \mathrm{~mm}^{2}$ ) of tumors inoculated into the mouse dorsal skin was $10.02 \pm 2.97,10.87 \pm 3.06$ and $5.44 \pm 2.48$ in Renca/Wild, Renca/LacZ and Renca/BAI1 tumors, respectively (Fig. 5b). The intratumoral blood volume was significantly $(\mathrm{p}<0.01)$ lower in the Renca/BAI1 tumor compared with the Renca/Wild or Renca/LacZ tumor. The blood content of normal skin was $1.75 \pm 0.88$.

Microvessels stained with CD31 were attenuated in the Renca/BAI1 tumors compared with the Renca/Wild and Renca/LacZ tumors (Fig. 6a). MVD was significantly lower in the Renca/BAI1 than in the Renca/Wild and Renca/LacZ tumors $(\mathrm{p}<0.05)$ (Fig. 6b).

Intratumor BAII viral vector injection. Tumor growth was inhibited significantly in the Renca/Wild tumors injected with the BAI1 viral vector compared with the tumors injected with PBS(-) from days 21 to 31 ( $\mathrm{p}=0.004$ on day 31) (Fig. 7). In addition, the same antitumor effect was found in the Renca/BAI1 tumor injected with the BAI1 viral vector compared with the tumors injected with PBS(-) from days 25 to 31 ( $\mathrm{p}=0.003$ on day 31$)$ (Fig. 7$)$.

\section{Discussion}

Tumor growth and metastasis depend on angiogenesis, and the suppression of new vascular formation is one of the pivotal strategies for inhibiting tumor progression $(1,2)$. A variety of pro- and anti-angiogenic molecules regulates new vascular formation. Angiogenesis is switched off when the effect of pro-angiogenic molecules is balanced by that of anti-angiogenic molecules. In contrast, a net balance tipped in favor of angiogenesis begins the angiogenesis process. Tumors themselves produce some angiogenesis activators and stimulate new vascular formation. Blocking angiogenesis could be an efficacious therapy for preventing tumor growth $(1,2)$. VEGF is one of the principal members of pro-angi-

a

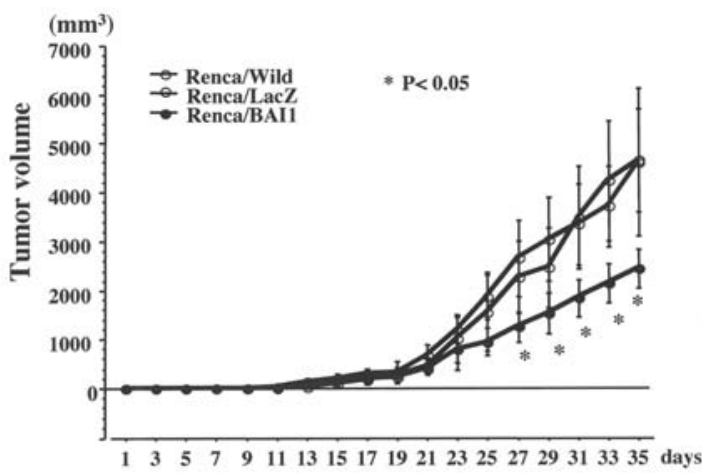

b

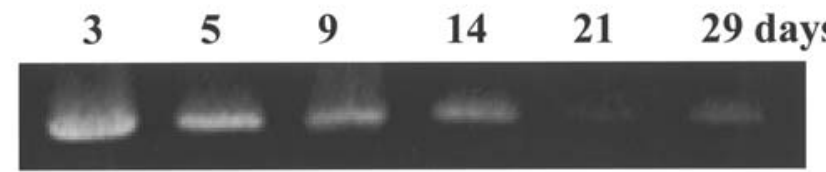

$453 \mathrm{bp}$

BAI1

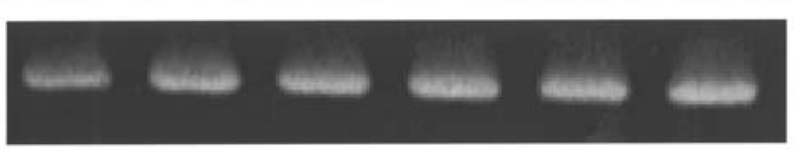

$452 b p$

G3PDH

Figure 4. In vivo tumor growth up to day 35 after the subcutaneous inoculation of tumor cells (a) and changes in BAI1 mRNA expression in the Renca/BAI1 tumors during the experimental period (b). The volume of the Renca/BAI1 tumor was significantly reduced compared with the Renca/Wild and Renca/LacZ tumors from day 27 onward $(\mathrm{p}<0.05)$. The expression of BAI1 mRNA was maintained until day 15 (b). On days 21 and 29 , BAI1 mRNA expression was attenuated. 
a

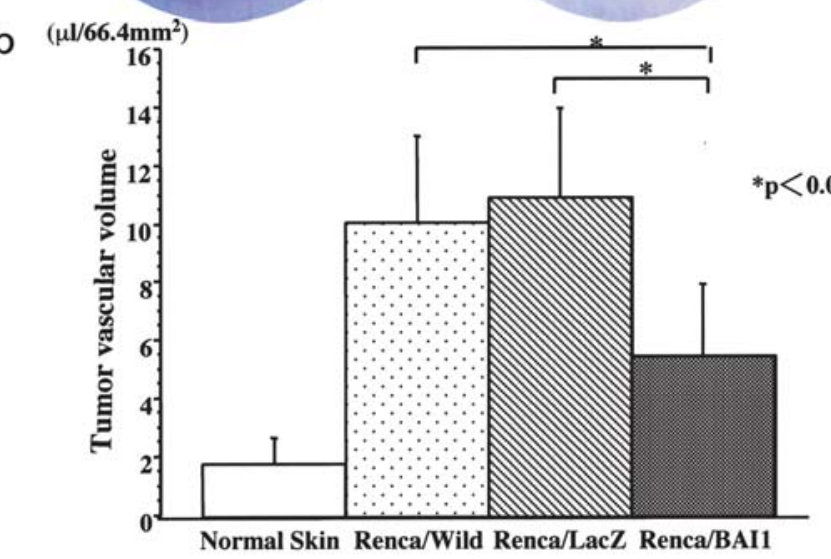

Figure 5. Tumor neovascularisation. (a) Macroscopic findings of tumor vascularization $72 \mathrm{~h}$ after subcutaneous inoculation. Angiogenesis is not promoted by Renca/BAI1 cells. In contrast, a stable vascular network is established by Renca/LacZ (arrow). (b) Intra-tumor blood volumes determined by the colorimetric angiogenesis assay method. A significantly lower blood volume was observed in the Renca/BAI1 tumor compared with the Renca/Wild and Renca/LacZ tumors $(\mathrm{p}<0.01)$.

ogenic molecules and the effectiveness of anti-VEGF therapy in inhibiting tumor growth has been reported (2). On the contrary, BAI that was originally isolated as a p53-target gene possessing an extracellular domain containing TSP type 1 repeats has been considered an inhibitor of angiogenesis (6). In vivo experiments have revealed that the transfection of the BAI1 gene into human pancreatic adenocarcinoma cells (Panc-1) results in suppressed tumor growth by inhibiting angiogenesis (10). In vitro studies have detected no difference in tumor growth between parental Panc-1 cells and BAI1 or LacZ transfectants, although an antiproliferative effect was found in human umbilical vein endothelial cells after the transfection of the BAI gene. In our in vivo study, the Renca/ BAI1 tumor implanted subcutaneously into female BAL/c mice demonstrated significantly suppressed growth, while similarly implanted Renca/Wild and Renca/LacZ tumors proliferated rapidly. Intratumor blood volume and MVD were significantly reduced in the Renca/BAI1 tumors compared with the Renca/Wild and Renca/LacZ tumors. Our in vitro study showed no difference in cell growth between Renca/BAIl and Renca/LacZ, consistent with the observation in human pancreatic adenocarcinoma cells (10). Since the BAI1 gene was expressed only in Renca/BAI1 cells and not in Renca/Wild or Renca/LacZ cells on RT-PCR, the suppression of tumor growth in Renca/BAI1 could be attributed to the reduction in vascular formation as a consequence of the BAI1 overexpression.

A peptide containing TSP type 1 repeats suppresses bFGF-induced neovascular formation (6). BAI-1 is a transmembrane protein and has five TSP type 1 repeats and an integrin binding site (RGD motif) in its extracellular domain (6). BAI-1 is proteolytically cleaved at a conserved G-proteincoupled receptor proteolytic cleavage site (GPS), releasing its $120 \mathrm{kDa}$ extracellular domain (14). This extracellular fragment has a potent anti-angiogenic action $(11,14)$. The definite mechanism for the anti-angiogenic effect of BAI1 is being studied, but recent study has reported that the secret extracellular domain of BAI1 exerts anti-proliferative action on the surrounding endothelial cells by blocking avß5 integrin partly through activating the caspase (11). In a previous in vitro study, a reduction in VEGF and matrix metalloproteinase-1 (MMP-1) expression has been demonstrated in a human pancreatic adenocarcinoma cell line (Panc-1) after BAI1, but not after LacZ transfer (10). The present study also showed a reduction in VEGF concentration in the supernatant of the Renca/BAI1 but not the Renca/LacZ cell culture. From these observations, BAI1 shows antiangiogenic activity not through a direct action on endothelial cells but through indirect effects by inhibiting other factors such as VEGF and MMP-1.

The expression of the BAI1 gene was initially considered to be specific to brain tissue (6). A lack of this gene is thought to be the cause of chaotic vascular proliferation in glioblastoma. However, BAI1 gene expression has subsequently been demonstrated in lung, gastric and colorectal cancer, suggesting that BAI1 plays an important role in the inhibition of angiogenesis associated with a variety of neoplasia other than cerebral tumors (7-9). A significantly lower MVD was demonstrated in lung cancers expressing BAI1 mRNA compared with those not expressing this mRNA (9). In colorectal cancers, the expression of BAI1 mRNA was significantly lower in malignant than in non-malignant colorectal tissues (7). BAI1 gene expression was inversely related to vascular invasion as well as metastasis, and also inversely correlated with vascular density. These two studies suggest that the expression of BAI1 can inhibit new vascular formation and/or tumor metastasis in lung and colorectal cancers. The expression of BAI1 mRNA was also reduced in gastric cancer (8). A significant prolonged survival period after gastrectomy has been demonstrated in patients with good BAI1 expression in the adjacent normal mucosa compared with those with poor BAI1 expression.

In the present study, the transferring of the BAI1 gene to mouse RCC Renca cells via an adenovirus vector resulted in inhibited tumor growth and suppressed tumor angiogenic activity in vivo, although tumor cell growth was not inhibited in vitro. Transfection of the human pancreatic adenocarcinoma cell line Panc-1 with the BAI gene also resulted in suppressed tumor growth in vivo but had no effect on in vitro cell growth (10). The inhibition of in vivo growth of the BAItransfected Panc-1 tumor was associated with the suppression of angiogenesis, as was also observed in the BAI1-transferred Renca tumors in the present study. As mentioned above, BAI1 could be one of the principal factors in the suppression 
a

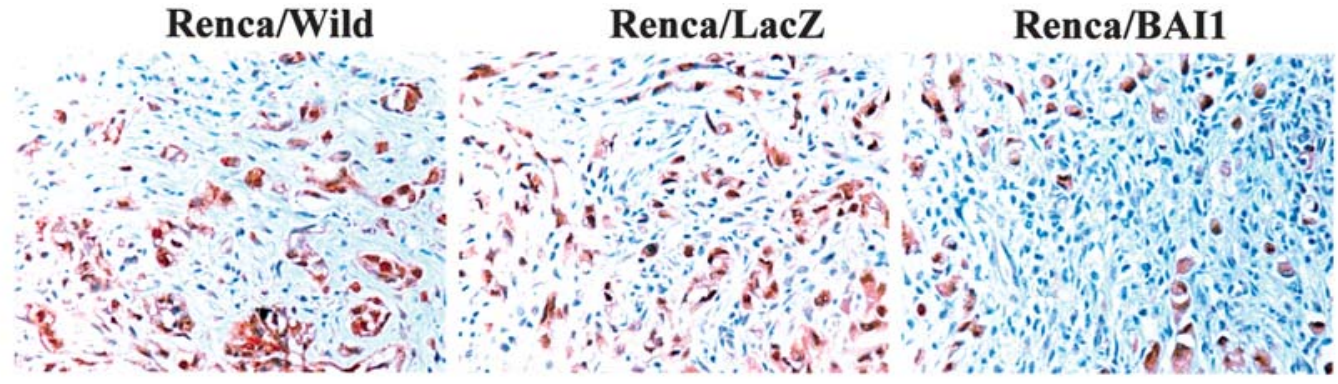

b

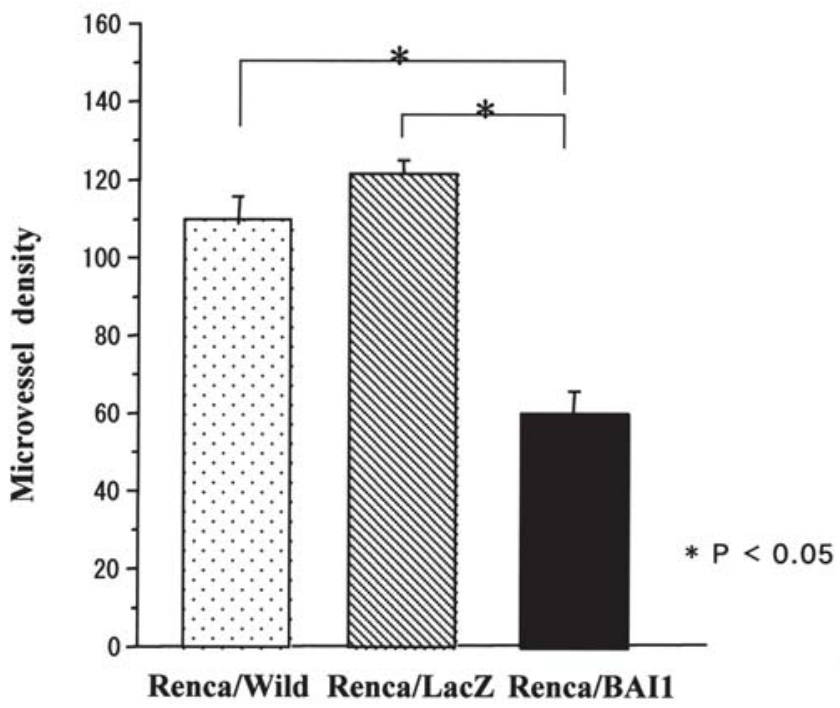

Figure 6. Immunostaining for CD31 (a) and microvessel density (MVD) (b) in Renca/Wild, Renca/LacZ and Renca/BAI1 tumors. The number of microvessels stained with CD31 was lower in the Renca/BAI1 than in the Renca/Wild and Renca/LacZ tumors (a). MVD was significantly lower in the Renca/BAI1 than in the Renca/Wild and Renca/LacZ tumors $(\mathrm{p}<0.05)$.

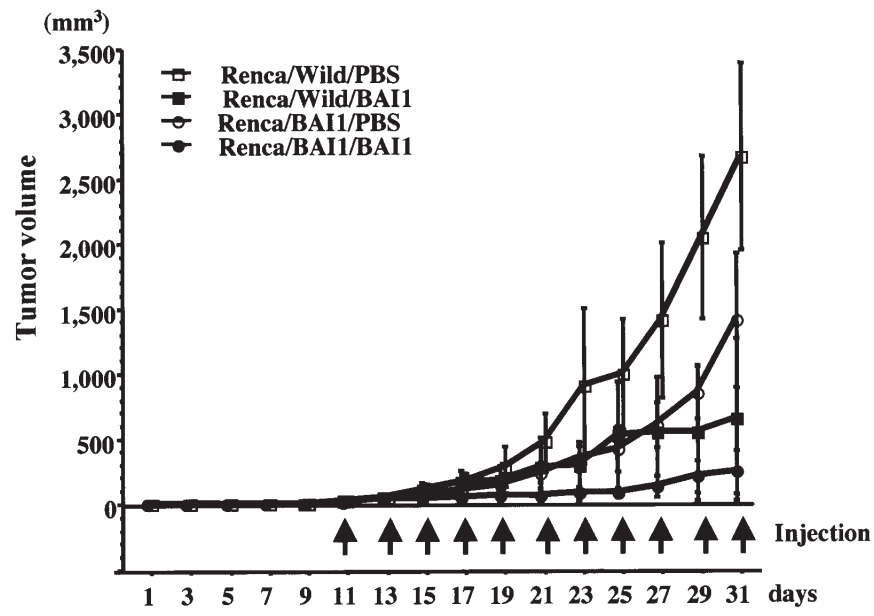

Figure 7. In vivo tumor growth after intratumoral injection of the BAI1 viral vector. Repeated BAI1 viral vector injection significantly suppresses tumor growth not only in the Renca/wild tumor but also in the Renca/BAI1 tumor, compared with the PBS(-) injection.

of tumor growth via the inhibition of angiogenesis and should be considered for gene therapy.

In order to examine the possibility of BAI1 as a candidate for gene therapy in RCC, we injected the BAI1 gene vector into subcutaneously implanted Renca/Wild and Renca/BAI1 tumors from the 11th day after implantation at 2-day intervals. The intratumor injection of the BAI1 gene vector significantly inhibited the growth of the Renca/Wild tumor compared with the PBS injection instead of the BAI1 gene. In addition, a significant reduction in the subcutaneously implanted Renca/BAI1 tumor was also achieved by the intratumor injection of the BAI1 gene, in comparison with the PBS injection. These observations could indicate the possibility of BAI gene therapy or the development of efficient drugs based on the extracellular fragment of BAI1 not only for RCC with decreased BAI1 expression but also for those with good BAI1 expression.

We investigated the antitumor effects of the BAI1 gene transfer into the murine RCC cell line, Renca, using replicationdeficient recombinant adenovirus vectors encoding the fulllength cDNA of BAI1. Significant suppression of both tumor growth and vascular formation was demonstrated in the Renca/BAI1 tumor compared with the Renca/Wild and Renca/LacZ tumors. The concentrations of VEGF were lower in the supernatant of the Renca/BAI1 cell culture compared with the Renca/Wild and Renca/LacZ cell cultures, suggesting that the suppression of VEGF production is involved in the anti-angiogenic effect of BAI1. Growth was inhibited in both the Renca/Wild and Renca/BAI1 tumors upon tumoral injection of a viral vector encoding BAI1, suggesting the possibility of the clinical application of this gene therapy. 


\section{Acknowledgements}

We deeply thank Professor Izumi Saito (Human Genomic Center, Institute of Medical Science, University of Tokyo) for AdexCA-LacZ, the viral vector containing the LacZ.

\section{References}

1. Calmelite P and Rakesh KJ: Angiogenesis in cancer and other disease. Nature 407: 249-257, 2000

2. Yancopoulos GD, Davis S, Gale NW, et al: Vascular-specific growth factors and blood vessel formation. Nature 407: 242-248, 2000.

3. Todd RF III, Garnic MB, George PC, et al: Phase I-II trial of methylglyoxal-bis guanylhydrazone (methyl-G) in the treatment of patients with metastatic renal adenocarcinoma. Cancer Treat Res 65: 17-20, 1981 .

4. De Kernion JB, Sarna G, Figlin R, et al: The treatment of renal cell carcinoma with human leukocyte $\alpha$-interferon. J Urol 130: 1063-1066, 1983

5. Campbell SC: Advances in angiogenesis res earch. Relevance to urological oncology. J Urol 158: 1663-1674, 1997.

6. Nishimori H, Shiratsuti T, Urano T, et al: A novel brain-specific p53-target gene, BAI1, containing thrombospondin type 1 repeats inhibits experimental angiogenesis. Oncogene 15: 2145$2150,1997$.

7. Fukusima Y, Oshika Y, Tsuchida T, et al: Brain-specific angiogenesis inhibitor 1 expression is inversely correlated with vascularity and distant metastasis of colorectal cancer. Int J Oncol 13: 967-970, 1998.
8. Heelee JI, Koh JT, Shin BA, et al: Comparative study of angiostatic and anti-invasive gene expression as prognostic factors in gastric cancer. Int J Oncol 18: 355-361, 2001.

9. Hatanaka H, Oshika Y, Abe Y, et al: Vascularization is decreased in pulmonary adenocarcinoma expressing brainspecific angiogenesis inhibitor1 (BAI1). Int J Mol Med 5: 181-183, 2000.

10. Duda DG, Sunamura M, Lozonschi L, et al: Overexpression of the p53-inducible brain-specific angiogenesis inhibitor 1 suppresses efficiently tumour angiogenesis. Br J Cancer 86: 490-496, 2002.

11. Koh JT, Kook H, Kee HJ, et al: Extracellular fragment of brainspecific angiogenesis inhibitor 1 suppresses endothelial cell proliferation by blocking avß5 integrin. Exp Cell Res 294: 172-184, 2004.

12. Nishizaki M, Fujiwara T, Tanida T, et al: Recombinant adenovirus expressing wild-type p53 is antiangiogenic: A proposed mechanism for bystander effect. Clin Cancer Res 5: 1015-1023,1999.

13. Fujioka T, Hasegawa M, Ogiu K, et al: Antitumor effects of angiogenesis inhibitor 0-(chloroacetyl-carbamoyl) fumagillol (TNP-470) agents in murin renal cell carcinoma. J Urol 155: $1775-1778,1996$

14. Kaur B, Brat DJ, Devi NS, et al: Vasculostatin, a proteolytic fragment of brain angiogenesis inhibitor 1, is an antiangiogenic and antitumorigenic factor. Oncogene 24: 3632-3642, 2005. 